\title{
Campomelic Dysplasia
}

National Cancer Institute

\section{Source}

National Cancer Institute. Campomelic Dysplasia. NCI Thesaurus. Code C84609.

A genetic congenital disorder characterized by bowing and angulation of the long bones.

It may be associated with other skeletal and extraskeletal defects. 